\title{
Characteristics of Pure Familial Pancreatic Cancer Families and Those with Additional Breast Cancer
}

\author{
Detlef K Bartsch $^{1 *}$, Elvira Matthäi ${ }^{1}$, Ioannis Mintziras ${ }^{1}$, Lutz Benedikt Böhm ${ }^{1}$, Norman Gercke ${ }^{1}$, Christian Bauer ${ }^{2}$, \\ Jens Figiel $^{3}$ and Emily P Slater ${ }^{1}$ \\ ${ }^{1}$ Department of Visceral, Thoracic and Vascular Surgery \\ ${ }^{2}$ Gastroenterology and Endocrinology \\ ${ }^{3}$ Radiology, Philipps University Marburg, Germany \\ *Corresponding author: Detlef K Bartsch, Philipps University of Marburg, Department of Visceral, Thoracic and Vascular Surgery, \\ Germany
}

\begin{abstract}
Familial pancreatic cancer (FPC) is a rare hereditary tumor syndrome. The large phenotypic and genotypic heterogeneity is not yet well-established. FPC families without (pure FPC) or with additional occurrence of breast cancer (FPC-breast) were analyzed regarding phenotype, genotype and the diagnostic yield of prospective screening for pancreatic ductal adenocarcinoma (PDAC). The total cohort of 227 FPC families included $85(38 \%)$ pure FPC and $70(30.7 \%)$ FPC-breast families. The proportions of affected family members with PDAC $(27.2 \%, 197 / 724$ vs. $18.3 \%, 177 / 962, \mathrm{p}<0.0001)$ and of 2 or more affected generations $(84.2 \%$ vs. $62.9 \%$, p $<0.05)$ were significantly higher in pure FPC families. In 48 (68.6\%) FPC-breast families additional tumor types occurred, most frequently colorectal cancer $(n=16,22.8 \%)$. Deleterious germline mutations (2 BRCA2, 1 PALB2) were detected in 3 of 53 (5.6\%) analyzed pure FPC and in 17 of 56 (30.4\%) analyzed FPC-breast families (3 BRCA1, 6 BRCA2, 3 PALB2, 3 CDKN2A, 2 ATM; p=0.001). Individuals at risk (IAR) from FPC-breast families participated significantly more often in a prospective PDAC screening program (54.4\% vs. 33.5\%; $\mathrm{p}=0.0001$ ) resulting in a diagnostic yield of $3.7 \%$ and $0 \%$ in FPC-breast and pure FPC families, respectively. The phenotypic and genotypic characteristics of pure FPC and FPC-breast families should be considered for the genetic counseling and management of these families.
\end{abstract}

Keywords: Familial Pancreatic Cancer; Mutations; Phenotype; Genotype

Abbreviations: FPC: Familial Pancreatic Cancer; PDAC: Pancreatic Ductal Adenocarcinoma; HBOC: Hereditary Breast and Ovarian Cancer; PanIN: Pancreatic Intraepithelial Neoplasia; IPMN: Intraductal Papillary Mucinous Neoplasia; AFL: Atypical Flat Lesions; IAR: Individual at Risk

\section{Introduction}

Pancreatic ductal adenocarcinoma (PDAC) is a challenging tumor entity with an increasing incidence and a dismal prognosis. The overall five-year-survival rate is less than $5 \%$, attributed to late clinical symptoms, low resection rates and poor response to radio- and chemotherapy [1]. One of the greatest risk factors for developing PDAC is a positive family history. A familial aggregation of PDAC is present in $1.9 \%$ to $6 \%$ of cases depending on the confirmation of the PDAC diagnosis by either only family history or by histopathology and medical reports $[2,3]$. An inherited predisposition to PDAC is potentially given in the so-called familial pancreatic cancer (FPC) families, which are characterized by two or more first-degree relatives with PDAC that do not fulfill the criteria for another inherited tumor syndrome [4, 5]. On the other hand several hereditary tumor predisposition syndromes such as PeutzJeghers-Syndrome (PJS) and hereditary breast and ovarian cancer (HBOC) might predispose to PDAC, although these syndromes are characterized by another clinical phenotype than PDAC [4, 5]. Tumor registries such as the North American National Familial Pancreatic Tumor Registry (NFPTR), the German National Case Collection of 
Familial Pancreatic Cancer (FaPaCa) and the European Registry of Hereditary Pancreatitis and Familial Pancreatic Cancer (EUROPAC) were established to investigate the phenotype and genotype of FPC families [6-8]. In the last years, studies focused mainly on the discovery of the underlying gene defects and the evaluation of diagnostic yield of prospective PDAC screening programs in individuals at risk (IAR) of such families [9-17]. Current sequencing and WES data suggest that FPC is genetically highly heterogeneous with no major predisposing gene [17]. The phenotypic variance of FPC families, however, is still not well-established. The current analysis was performed to analyze the phenotype and genotype and the diagnostic yield of prospective PDAC screening in pure FPC and FPC-breast cancer families.

\section{Materials and Methods}

The FaPaCa registry is a national case collection for familial pancreatic cancer families in Germany funded by the Deutsche Krebshilfe in 1999 [7-18]. Families with two or more first-degree relatives with a confirmed diagnosis of PDAC and without evidence of any other inherited tumor syndrome were collected. The current report analyzed the genotype and phenotype of FPC families with the occurrence of only PDAC (pure FPC families) and FPC families with the additional occurrence of breast cancer (FPC-breast) families. Members from FPC families were recruited between July 1999 and July 2019 by direct referral via their physicians or by personal contact to the FaPaCa study-office based on information about the study, e.g. via the internet (http://www.fapaca.de). All eligible persons and families were initially genetically counseled and a three-generation family pedigree was constructed. All PDAC diagnoses were confirmed by review of medical records, deaths certificates and by examination of the pathology slides when available. All patients with PDAC of FPC families with available blood samples who gave their informed consent were subjected to mutation analysis of the potential predisposing PDAC genes ATM, BRCA1/2, CDKN2A, PALB2, PLLD and CHEK2 as described previously [12-18]. In addition, whole exome sequencing (WES) was performed in 7 of those families (Slater et al., submitted). If a deleterious germline mutation was identified in the index patient, predictive genetic testing of this mutation was offered to all family members after genetic counseling. The result of the predictive testing was explained to the family members during another interdisciplinary counseling, involving a geneticist, a psychologist, a surgeon and gastroenterologist. Individuals at risk (IAR) older than 18 years were encouraged to participate in a prospective screening program that was conducted at our institution. Firstdegree relatives of an affected patient of a FPC family and members of a FPC-family carrying a predisposing mutation such as BRCA2, independent of the degree of relationship, were classified as IAR. The screening started at age 40 years until 2016 and thereafter at age 50 years or 10 years before the earliest age of onset of PDAC in the family, whichever was first [19]. The screening program included an annual physical examination, determination of serum HbA1c, amylase, GOT, GPT, bilirubin and CA19-9, and imaging with magnetic resonance imaging plus MRCP and endosonography as described previously [19]. The screening program was restricted to mutation carriers, if the underlying gene defect was known in the family. Resection specimens were analyzed by experienced pathologists with special regard to the presence of PDAC, pancreatic intraepithelial neoplasia (PanIN), intraductal papillary mucinous neoplasia (IPMN) and atypical flat lesions (AFL). Previous screening results of some IAR have been already published [10, 18-20]. The FaPaCa registry, including the genetic analyses and the screening program, was approved by the Ethics Committee of the PhilippsUniversity of Marburg (36/1997, last amendment 9/2010) and all participants provided written informed consent. Descriptive statistics of the relatives who enrolled were compiled. Variables included age, gender, number of relatives with PDAC, earliest age of onset in the family and underlying germline mutations. The age of diagnosis of PDAC was retrieved from the 3-generation pedigrees and early age of onset was defined as the occurrence of PDAC prior to the age of 50 years in a family. Significant lesions were defined as the presence of histologically verified PDAC, PanIN3 or IPMN with high-grade dysplasia. Potentially relevant lesions were defined as histologically verified multifocal PanIN2 lesions with/without BDIPMN with low grade dysplasia and/or atypical flat lesions (AFL). The chi-square, Fisher's exact test, t test and Wilcoxon rank sum test were performed for categorical and numerical variables, where appropriate, to compare patient characteristics. Two-tailed $\mathrm{p}$ values $<0.05$ were considered to be statistically significant. Analyses were performed using Prism 6 for Mac OS X from GraphPad Software, Inc.

\section{Results}

Of 227 verified FPC families, 85 (37.4\%) revealed only PDAC and $70(30.8 \%)$ families showed both PDAC and breast cancer. The proportion of affected family members with PDAC was significantly higher in pure FPC families $(27.2 \%, 197 / 724)$ compared to all FPCbreast families $(18.3 \%, 177 / 962, \mathrm{p}<0.0001)$. This also holds true when comparing FPC-breast families with or without detected predisposing germline mutations. In the 17 FPC breast families with a germline mutation the PDAC rate was $18.3 \%$ (49 of 267 family members) compared to $16.4 \%$ (90 of 550 family members) in the 39 families without mutations. Twenty (24\%) pure FPC families had 3 or more affected first-degree relatives with PDAC compared to $26(37.1 \%)$ of the FPC-breast families $(p=0.08)$. The proportion of 2 or more affected generations, however, was significantly higher in pure FPC families than in FPC-breast families (84.2\% vs. $62.9 \%, \mathrm{p}<0.05)$. The rate of females affected with PDAC tended to be lower in pure FPC $(48 \%, 94 / 197)$ than in FPCbreast families $(56 \%, 100 / 177, \mathrm{p}=0.08)$. The median age of PDAC diagnosis (63 vs. 65 years) and the rate of families with early age of PDAC onset $<50$ years ( $23.5 \%$ vs $21.4 \%$ ) were not significantly different between family groups (Table 1). In the 70 FPC-breast 
families a total of 113 (1-6 per family) breast cancers occurred, all but one in female patients. The mean rate of breast cancer cases per family was 1.6 ( $\mathrm{SD}=1.207$; $\mathrm{SEM}=0.1443)$. At inclusion none of these 70 families fulfilled the criteria for HBOC $[21,22]$, but this was the case for six $6(8.6 \%)$ families due to the additional occurrence of breast/ovarian cancer cases after a median follow-up of 7 years. In 19 families 23 (23\%) female patients developed PDAC and breast cancer, synchronous and metachronous. The median age of breast cancer diagnosis was 55 (range 24-93) years, 31 (27.4\%) patients had a premenopausal ( $<50$ years) diagnosis. Five $(3.6 \%)$ female patients developed bilateral breast cancer. In 48 (68.6\%) FPCbreast family's tumor types in addition to PDAC and breast cancer also occurred. The most frequent additional cancers were colon cancer in 16 (22.8\%), lung cancer in 10 (14.3\%), prostate cancer and malignant melanoma in $8(11.4 \%)$ families each, respectively (Table 1). The characteristics of pure FPC families and FPC-breast families are summarized in (Table 1). Representative pure FPC and FPC-breast families are shown in (Figures 1,2).

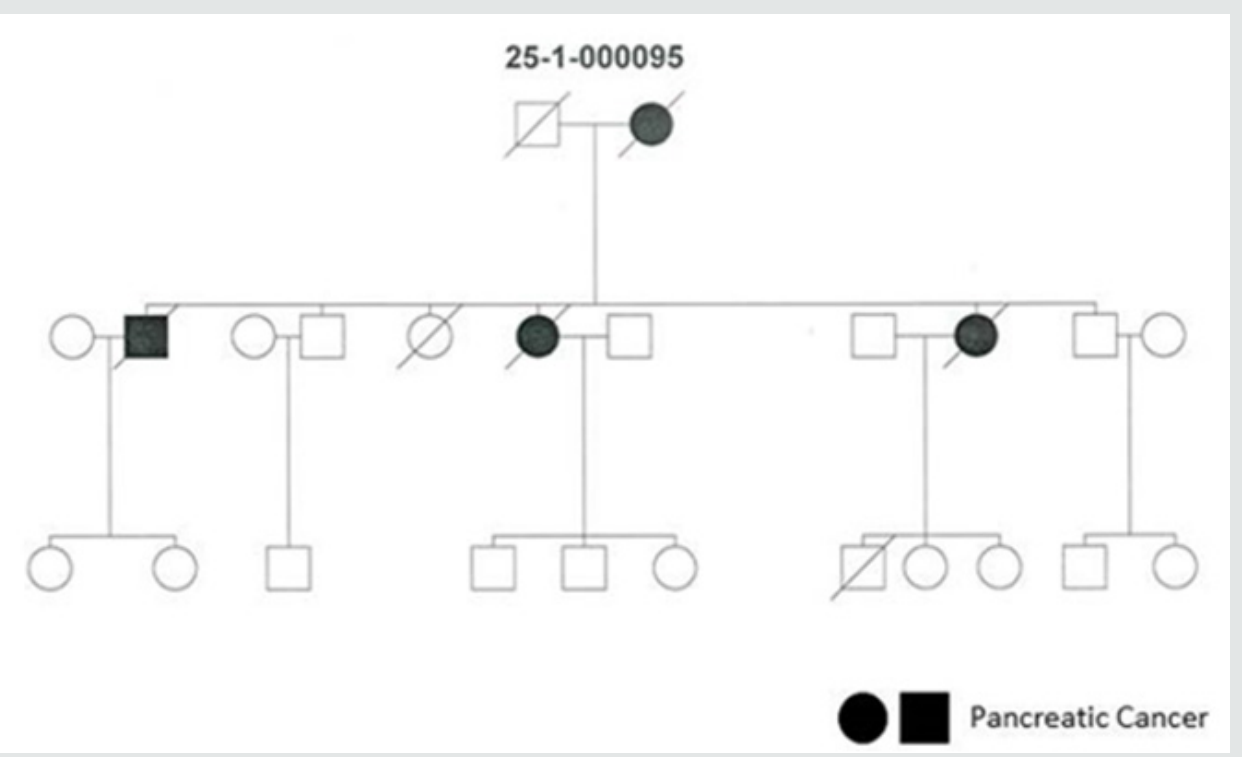

Figure 1: Pedigree of a representative "pure" FPC family.

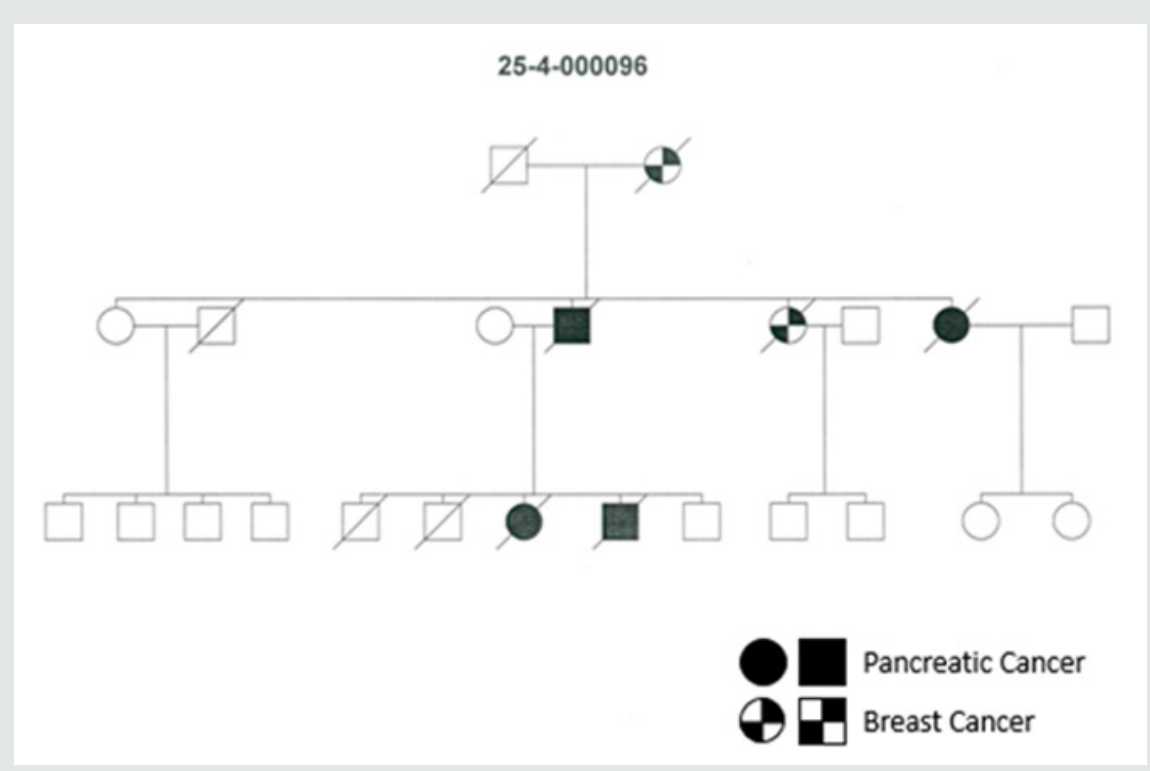

Figure 2: Pedigree of a representative FPC-breast family. 
Table 1: Characteristics of "pure" FPC and FPC-breast families.

\begin{tabular}{|c|c|c|c|}
\hline Characteristic & pure FPC $(n=85)$ & FPC with Breast Cancer $(n=70)$ & p-value \\
\hline Total Number Family Members & 724 & 962 & \\
\hline Total Number PDAC Cases & $197(27.2 \%)$ & $177(18.3 \%)$ & $<0.0001$ \\
\hline \multicolumn{4}{|l|}{ FDR with PDAC/family } \\
\hline 2 & $65(76 \%)$ & $44(62.8 \%)$ & ns (0.0781) \\
\hline 3 & $14(16 \%)$ & $17(24.3 \%)$ & ns \\
\hline$>3$ & $6(8 \%)$ & $9(12.8 \%)$ & ns \\
\hline 1 Affected Generation with PDAC & $13(15.2 \%)$ & $26(37.1 \%)$ & 0.0027 \\
\hline 2 Affected Generations with PDAC & $67(79.0 \%)$ & $41(58.7 \%)$ & 0.0083 \\
\hline 3 Affected Generation with PDAC & $5(5.8 \%)$ & $3(4.2 \%)$ & ns \\
\hline median age at Dx PDAC & 63 (35 to 91) & 65 (32 to 86$)$ & ns \\
\hline $\begin{array}{l}\text { families with early age of onset of } \\
\text { PDAC ( }<50 \text { yrs. })\end{array}$ & $20(23.5 \%)$ & $15(21.4 \%)$ & ns \\
\hline Female with PDAC & $94 / 197(48 \%)$ & $100 / 177(56 \%)$ & ns (0.0978) \\
\hline FDR with breast cancer/family & & 7 & \\
\hline 1 & - & 48 & \\
\hline 2 & - & 9 & \\
\hline 3 or more & - & 6 & \\
\hline female/male with BC & - & $112 / 1$ & \\
\hline $\begin{array}{l}\text { Families Fulfilling Criteria of } \\
\text { HBOC1 }\end{array}$ & 0 & 6 & \\
\hline Age Breast Cancer, Median & - & 55 (24-93) & \\
\hline Patients with Premenopausal BC & & $31(27.6 \%)$ & \\
\hline patients with PDAC and BC & 0 & 23 of 19 families & \\
\hline families with other tumor types & 0 & $48(68.5 \%)$ & \\
\hline \multirow{6}{*}{ other cancer types in the family } & \multirow{6}{*}{ - } & colon (16 families) & \\
\hline & & melanoma (8 families) & \\
\hline & & lung (10 families) & \\
\hline & & ovarian (6 families), & \\
\hline & & endometrium (5 families) & \\
\hline & & prostate (8 families) & \\
\hline $\begin{array}{l}\text { families with identified deleterious } \\
\text { germline mutations }\end{array}$ & $3 / 53(5.6 \%)$ & $17 / 56(30.4 \%)$ & 0.0012 \\
\hline mutated genes (n) & BRCA2 (2), PALB2 (1) & $\begin{array}{l}\text { BRCA2 (6), BRCA1 (3), CDKN2A (3), } \\
\text { PALB2 (3), ATM (2), }\end{array}$ & \\
\hline
\end{tabular}

Mutation analysis complemented by WES sequencing of 7 families identified deleterious gene alterations in known predisposing PDAC genes, such as BRCA2 and PALB2, in 3 of 53 (5.6\%) analyzed pure FPC-families and 17 of 56 (30.4\%) analyzed FPC-breast families $(\mathrm{p}=0.001)$. In pure FPC families only BRCA2 and PALB2 were altered, whereas in the FPC-breast families several genes, including BRCA1/2, CDKN2A, PALB2 and ATM were mutated (Table 2). The most frequently mutated gene was BRCA2 in 8 of $20(40 \%)$ families with mutations. In all pure FPC and FPC-breast families the identified gene defect co-segregated with the disease. Variants of unknown significance in several genes such as ACAD9,
KCNJ12, KMTC2, NOTCH2NL and SUFU were identified in 5 of 7 families investigated with WES. These gene alterations did not always segregate with PDAC disease in the respective families and are classified in ClinVar as variants of unknown significance.

Individuals at risk (IAR) from FPC-breast families (135 of 248, $54.4 \%$ ) participated significantly more often in a recommended screening program than IAR from pure FPC families (74 of 221, $33.5 \%$; $\mathrm{p}<0.0001$ ). The median follow-up was 34 months in the IAR of pure FPC and 51 months in IAR of FPC-breast families ( $p=0.0366$ ). IAR of pure FPC and FPC-breast families were comparable regarding gender distribution, smoking habits, the presence of diabetes 
mellitus and history of pancreatitis, respectively (Table 3). A total of 904 examination visits (median 3 ranges 1-16) were performed in the 209 IAR over the period. On imaging small $(<10 \mathrm{~mm})$ cystic lesions, most likely BD-IPMN, were detected in $68.9 \%$ and $63.7 \%$ of IAR form pure FPC and FPC-breast families. Imaging detected "worrisome" lesions in 2 (2.7\%) IAR from pure FPC and 10 IAR (7.5\%) from FPC-breast families $(p=0.21)$. These 12 IAR underwent pancreatic resections and significant lesions such as PDAC or PanIN3 were detected in 5 (3.7\%) of the IAR from FPC-breast families.
None of these IAR was a carrier of a PDAC-predisposing germline mutation. The other 5 IAR from the FPC-breast families, including 2 germline mutation carriers, revealed potentially relevant multifocal PanIN2 lesions and/or IPMN with moderate dysplasia, whereas both operated IAR from pure FPC families had non-relevant lesions such serous cystadenoma and focal fibrosis (Table 3). Thus, the diagnostic yield of the prospective PDAC screening was $0 \%$ and $3.7 \%$ in pure FPC and FPC-breast families according to the strict criteria of an international consensus conference $[5,22]$.

Table 2: Deleterious germline mutations in pure FPC and FPC-breast families.

\begin{tabular}{|c|c|c|c|c|}
\hline Family type & Family- ID & Gene mutated & Alteration & other associated cancer \\
\hline Pure FPC & 02-8-0027 & BRCA2 & c.6444dup; p. (Ile2149Tyrfs*2) & - \\
\hline Pure FPC & $25-6-239$ & BRCA2 & c.2899_2900del; p. (Leu967Argfs*14) & - \\
\hline Pure FPC & $25-2-102$ & PALB2 & c.509_510del; p. (Arg170Ilefs*14) & - \\
\hline FPC-Breast & $09-3-0026$ & BRCA2 & $\begin{array}{c}\text { c.9227G>A; p. (Gly3076Glu) } \\
\text { c.10095delins11; p. (Ser3366Asnfs*4) }\end{array}$ & - \\
\hline FPC-Breast & $25-7-1$ & BRCA2 & c.6591_6592del; p. (Glu2198Asnfs*4) & $\begin{array}{l}\text { colon (1), prostate (2), } \\
\text { gastric (1) }\end{array}$ \\
\hline FPC-Breast & $25-8-142$ & BRCA2 & c.3847_3848delGT; p. (Val1283Lysfs*2) & Ovarian (1) \\
\hline FPC-Breast & $25-1-171$ & BRCA2 & c.1813del; p. (Ile605Tyrfs*9) & - \\
\hline FPC-Breast & $25-5-208$ & BRCA2 & c.3708dup; p. (Ala1237Serfs*6) & - \\
\hline FPC-Breast & $25-3-232$ & BRCA2 & c.3847_3848delGT; p. (Val1283Lysfs*2) & ovarian (1), lung (1) \\
\hline FPC-Breast & $25-6-116$ & BRCA1 & c.5278-1G>T splice variant (20i) & ovarian (1) \\
\hline FPC-Breast & $25-7-166$ & BRCA1 & c.5383dup; p. (Leu1795Profs*35) & $\begin{array}{c}\text { ovarian (1), hepatocellular } \\
\text { (1) }\end{array}$ \\
\hline FPC-Breast & $25-7-188$ & BRCA1 & c.2702_2703del; p. (Phe901*) & colorectal (2), prostate (1) \\
\hline FPC-Breast & $25-9-56$ & PALB2 & c. 1240 C >T; p. $\left(\operatorname{Arg} 414^{*}\right)$ & Colon (1) \\
\hline FPC-Breast & $25-4-149$ & PALB2 & c.3362del; p. (Gly1121Valfs*3) & cholangio (1), leukemia (1) \\
\hline FPC-Breast & $25-2-199$ & PALB2 & c.509_510del; p. (Arg170Ilefs*14) & - \\
\hline FPC-Breast & $25-9-118$ & CDKN2A & c.132C>A; p. (Tyr44*) & malignant melanoma (2) \\
\hline FPC-Breast & $25-2-129$ & CDKN2A & c.301G>T; p. (Gly101Trp) & basal cell (1), prostate (1) \\
\hline FPC-Breast & $25-9-200$ & CDKN2A & c.301G>T; p. (Gly101Trp) & basal cell (1), testicular (1) \\
\hline FPC-Breast & $02-5-0382$ & ATM & c.5385G>T; p. (Trp1795Cys) & $\begin{array}{l}\text { acute lymphoblastic } \\
\text { leukemia (1) }\end{array}$ \\
\hline FPC-Breast & $25-5-187$ & ATM & c.3802delG; p. (Val1268*) & - \\
\hline
\end{tabular}

Table 3: Characteristics of IAR and results of PDAC screening.

\begin{tabular}{|c|c|c|c|}
\hline & Pure FPC Families & FPC-breast cancer families & p-value \\
\hline Families & 85 & 70 & $\mathrm{p}=0.0338$ \\
\hline Families in Screening & $42(49 \%)$ & $47(67 \%)$ & $\mathrm{p}<0.0001$ \\
\hline IARs in controlled screening & $74 / 221(33.5 \%)$ & $135 / 248(54.4 \%)$ & $\mathrm{p}=0.0366$ \\
\hline median Follow-up (months) & $34(0$ to 216$)$ & $51.5(0$ to 188$)$ & $\mathrm{p}=0.0262$ \\
\hline IAR with at least 5 year follow-up & $21(28 \%)$ & $60(44.4 \%)$ & $\mathrm{p}=0.0195$ \\
\hline median age of IAR & $52.5($ range $31-70)$ & $56.5($ range $35-77)$ & $\mathrm{p}=0.0078$ \\
\hline $\begin{array}{c}\text { IAR with deleterious germline } \\
\text { mutations }\end{array}$ & $2(2.7 \%)^{1}$ & $19(14.1 \%)^{2}$ & $\mathrm{~ns}$ \\
\hline Female IAR & $40(54 \%)$ & $89(66 \%)$ & $\mathrm{ns}$ \\
\hline IAR who smoked & $27(36.5 \%)$ & $51(37.8 \%)$ & $\mathrm{ns}$ \\
\hline IAR with diabetes & $4(5.4 \%)$ & $6(4.4 \%)$ & $\mathrm{ns}$ \\
\hline IAR with pancreatitis & $2(2.7 \%)$ & $6(4.4 \%)$ & \\
\hline
\end{tabular}




\begin{tabular}{|c|c|c|c|}
\hline $\begin{array}{c}\text { IAR with cystic lesions on } \\
\text { imaging }\end{array}$ & $51(68.9 \%)$ & $86(63.7 \%)$ & ns \\
\hline IAR with solid lesions on imaging & 0 & 2 & ns \\
\hline $\begin{array}{c}\text { IAR with worrisome lesions on } \\
\text { imaging }\end{array}$ & 2 & 10 & ns \\
\hline IAR who underwent surgery & $2(2.7 \%)$ & $10(7.5 \%)$ & ns \\
\hline total pancreatectomy & 0 & 7 & \\
\hline PPPD & 0 & 1 & \\
\hline PLR & 2 & 2 & \\
\hline \multicolumn{4}{|l|}{ Histology } \\
\hline PDAC & 0 & 2 (рT2, N0; pT3, N0) & \\
\hline PanIN3 & 0 & 3 & \\
\hline PanIN2 or IPMN with LGD & 0 & 5 & \\
\hline significant lesions $^{3}$ & $0(\%)$ & $5(3.7 \%)$ & \\
\hline non-relevant lesions & 2 & 0 & \\
\hline
\end{tabular}

\section{Discussion}

Despite several new molecular insights into the pathogenesis of hereditary forms of cancer, its translation into the clinical setting remains still somehow problematic. A comprehensive history of cancer in a family is still one milestone to establish a potentially hereditary cancer syndrome. The family history, however, has been insufficiently recorded in many patients' medical records, thereby compromising its clinical significance [24]. This is especially true for FPC families, since the phenotype is highly heterogeneous and affected patients die fast due to the aggressive disease. FPC can be mainly divided into two groups, namely pure PC families and those associated with other tumor types [4]. The most frequent other tumor type associated with FPC in the FaPaCa registry is breast cancer, in almost one third (30.7\%) of FPC families. Therefore, we undertook a detailed analysis of the phenotype and genotype of pure FPC families and FPC with an additional occurrence of breast cancer. Initially, none of these families fulfilled the criteria for HBOC $[21,22]$, but after a median follow-up of 7 years this was the case for $6(8.6 \%)$ families due to the new development of additional breast and/or ovarian cancer cases. It has been previously reported that some HBOC families are associated with an increased risk for PDAC. A retrospective analysis of 5143 Italian family trees with breast and/ or ovarian cancer, for example, showed that 392 (7.6\%) families also had cases of PDAC [25]. It has also been postulated that the risk of PDAC is especially increased in those 25 to $30 \%$ of HBOC families who are associated with BRCA1 or BRCA2 germline mutations [25]. Lynch et al. reported that 213 (3.7\%) of 5742 families with a BRCA1 mutation and 138 (6.1\%) of 2269 families with a BRCA2 mutation had a case of PDAC [26]. A study by the Breast Cancer Linkage Consortium (BCLC) yielded for female BRCA1 mutation carriers a 2.3-fold risk and for female BRCA2 mutation carriers a 3.5-fold risk for the development of PDAC [27]. From another viewpoint, it has been reported that a familial accumulation of PDAC might be associated with the occurrence of breast and/or ovarian cancer.
A mortality analysis of the National Familial Pancreatic Tumor Registry probands, which included over 200,000 person-years of follow-up from 8564 first-degree relatives of PDAC probands, found that relatives of FPC probands had a significantly increased risk of dying from breast (wSMR 1.66, 95\% CI 1.15-2.34), ovarian (wSMR $2.05,95 \%$ CI 1.10-3.49), and bile duct cancers (wSMR 2.89, 95\% CI 1.04-6.39) [28]. Analysis of the familial aggregation of PDAC with other malignancies using the updated Swedish Family-Cancer Database with more than 11.5 million individuals disclosed that a significantly increased the risk of PDAC was associated with earlyonset breast cancer in siblings [29].

Based on the present analysis one can postulate some phenotypic differences between pure FPC and FPC-breast families. The proportion of affected family members with PDAC $(27.2 \%$ vs. $18.3 \%, \mathrm{p}<0.0001$ ) and the involvement of 2 or more generations was significantly higher in pure FPC families than in FPC-breast families (84.2\% vs. 62.9\%, p<0.05). The rate of females affected with PDAC tended to be higher in FPC-breast than in pure FPC families (56\% vs. $48 \%, \mathrm{p}=0.0978$ ). A previous analysis of 317 BRCA $1 / 2$ mutated HBOC families with 351 cases of PDAC, however, revealed a slightly higher risk in males (58\% vs. 46\%) [26]. The median age of PDAC diagnosis ( 63 vs. 65 years) and the rate of families with early age of PDAC onset $<50$ years $(23.5 \%$ vs $21.4 \%$ ) were not significantly different between family groups (Table 1), which is in line with previous reports on pure FPC families and BRCA1/BRCA2 families with the occurrence of PDAC $[26,27]$.

Comparing the FPC-breast families with the reported characteristics of HBOC families one can also note some phenotypical differences. The PDAC rate in FPC-breast families (18\%) appears comparably high to HBOC families with 1.5 to $8 \%$ [24, 25]. In the FPC-breast families the rate of breast cancer cases with mean 1.6 cases per family was lower than in German HBOC families [30, 31]. The median age of onset of breast cancer (55 vs. 49 years) was 
older and the proportion of women with premenopausal onset of breast cancer ( $27.4 \%$ vs. $45 \%$ ) was also lower than those reported for German HBOC families [30]. It is of note, that $68.5 \%$ of analyzed FPC-breast families showed additional cancers, including colorectal cancer $(22.8 \%)$ and ovarian cancer (8.6\%).

Recentadvances in sequencingtechnology provided an unbiased way to search for the genes underlying disease susceptibility in FPC. Using this approach BRCA1/2, CDKN2A, PALB2 and ATM were identified as FPC susceptibility genes, together explaining about $8 \%-15 \%$ of FPC cases $[17,18,32]$. A previous whole genome sequencing study of 638 patients from 593 American FPC kindreds demonstrated that the genetic underpinning of inherited pancreatic cancer is highly heterogeneous [17]. This analysis supported the role of the known FPC susceptibility BRCA2, CDKN2A, PALB2 and ATM, and identified a few novel candidate genes such as BUB1B and FANCC harboring rare, deleterious germline variants. Interestingly, many of these candidate genes are involved in processes regulating DNA repair or chromosomal stability.

The genetic basis underlying FPC susceptibility, however, remains still unknown in $80 \%$ to $90 \%$ of patients with FPC. In the present study a predisposing gene defect could be identified in the susceptibility genes BRCA1, BRCA2, CDKN2A, ATM and PALB2 in 17 (15.5\%) of 109 analyzed FPC families. As in previous studies BRCA2 was the most frequently mutated gene 8 (7.3\%). It is of note, however, that the prevalence of deleterious mutations was significantly higher in FPC-breast (30.4\%) than in pure FPCfamilies (5.6\%, p=0.001). ATM, BRCA1 and CDKN2A mutations were only detected in FPC-breast families. A previous Italian study postulated a BRCA2 cluster region within c.7180 and c.8248, which might be associated with the occurrence of PDAC [25]. We cannot confirm this observation, since all of the 8 BRCA2 mutations were located outside of this region.

Knowledge of the genes responsible for FPC susceptibility is important for a number of reasons. One is that early detection can be targeted to mutation carriers and pancreatic neoplasms can be detected at an earlier stage, when therapeutic interventions with curative intent are still possible. A recent expert consensus conference recommends pancreatic screening under boardapproved protocols for IAR from FPC families [5, 23]. Various screening programs reported a diagnostic yield of PDAC screening between 1.9 and 7\%, when defining high grade dysplasia or stage I PDAC as true success of screening $[9,10,19]$. In the present study it was obvious that IAR from FPC-breast families participated significantly more often in the recommended screening than IAR from pure FPC families (33.5\% vs. 54.4\%, p<0.0001). The reasons for this remain speculative and are probably multifactorial. The diagnostic yield of the screening program itself appears to be more efficient in FPC-breast families compared to pure FPC families (3.7\% vs. $0 \%$ ), although the prospective follow-up of less than 5 years is too short to draw any definitive conclusions. A recent North American study [33] reported that the cumulative incidence of PDAC and high grade dysplasia was significantly higher in the IAR group with predisposing germline mutations compared to those IAR without (RR 2.85, 95\% CI 1.0-8.18, p=0.05). We can neither confirm nor reject this observation due to the limited number of mutation carriers $(n=21)$ participating in screening.

\section{Conclusion}

In summary, there seems to be a distinct FPC-breast family phenotype, which might be associated with other solid cancers such as colon cancer and has at least a $30 \%$ prevalence of predisposing germline mutations in known susceptibility genes BRCA1/2, PALB2, CDKN2A and ATM. IAR of these families have a comparably high motivation to participate in PDAC screening, which in short term appears to be more effective than in pure FPC families. These data should be considered for the counseling and management of these families.

\section{Acknowledgements}

We are grateful to all FPC families for participating in the registry. We thank A. Ramaswamy, Marburg, and Günter Klöppel, Munich, for the pathological examination of the resection specimens. This study was supported by the Deutsche Krebshilfe, grant number 111092, and a generous donation from the GAUFF-Foundation.

\section{Conflict of interest}

None of the authors has competing financial interests nor other conflicts of interest.

\section{References}

1. Quante A, Ming C, Rottmann M, Engel J, Boeck S, et al. (2016) Projections of cancer incidence and cancer-related deaths in Germany by 2020 and 2030. Cancer Med 5(9): 2649-2656.

2. Bartsch DK, Kress R, Sina-Frey M, Grützmann R, Gerdes B, et al. (2004) Prevalence of familial pancreatic cancer in Germany. Int J Cancer $110(6), 902-906$.

3. Hemminki K, Li X (2003) Familial and second primary pancreatic cancers: a nationwide epidemiologic study from Sweden. Int J Cancer 103(4): 525-530.

4. Bartsch DK, Gress TM, Langer P (2012) Familial pancreatic cancer current knowledge. Nat Rev Gastroenterol Hepatol 9(8): 445-553.

5. Canto MI, Harinck F, Hruban RH, George Johan Offerhaus, Jan-Werner Poley, et al. (2013) International Cancer of Pancreas Screening (CAPS) Consortium International Cancer of the Pancreas Screening (CAPS) Consortium summit on the management of patients with increased risk for familial pancreatic cancer. Gut 62(3): 339-347.

6. Tersmette AC, Petersen GM, Offerhaus GJ, Falatko FC, Brune KA, et al. (2001) Increased risk of incident pancreatic cancer among firstdegree relatives of patients with familial pancreatic cancer. Clin Cancer Res 7(3): 738-744.

7. Bartsch DK, Sina-Frey M, Ziegler A, Hahn SA, Przypadlo E, et al. (2001) Update of familial pancreatic cancer in Germany. Pancreatology 1(5): 510-516. 
8. Applebaum SE, Kant JA, Whitcomb DC, Ellis IH (2000) Genetic testing counselling, laboratory, and regulatory issues and the EUROPAC protocol for ethical research in multicenter studies of inherited pancreatic diseases. Med Clin North Am 84(3): 575-588.

9. Canto MI, Almario JA, Schulick RD, Yeo CJ, Klein A, et al. (2018) Risk of neoplastic progression in individuals at high risk for pancreatic cancer undergoing long-term surveillance. Gastroenterology 155(3): 740-751.

10. Vasen H, Ibrahim I, Ponce CG, Slater EP, Matthäi E, et al. (2016) Benefit of surveillance for pancreatic cancer in high-risk individuals: outcome of long-term prospective follow-up studies from three european expert centers. J Clin Oncol 34(17): 2010-2019.

11. Corral JE, Mareth KF, Riegert-Johnson DL, Das A, Wallace MB, et al. (2019) Diagnostic yield from screening asymptomatic individuals at high risk for pancreatic cancer: a meta-analysis of cohort studies. Clin Gastroenterol Hepatol 17(1): 41-53.

12. Hahn SA, Greenhalf B, Ellis I, Sina-Frey M, Rieder H, et al. (2003) BRCA2 germline mutations in familial pancreatic carcinoma. J Natl Cancer Inst 95(3): 214-221.

13. Murphy KM, Brune KA, Griffin C, Sollenberger JE, Petersen GM, et al. (2002) Evaluation of candidate genes MAP2K4, MADH4, ACVR1B, and BRCA2 in familial pancreatic cancer: deleterious BRCA2 mutations in 17\%. Cancer Res 62(13): 3789-3793.

14. Bartsch DK, Sina-Frey M, Lang S, Wild A, Gerdes B, et al. (2002) CDKN2A germline mutations in familial pancreatic cancer. Ann Surg 236(6): 730-737.

15. Slater EP, Langer P, Niemczyk E, Strauch K, Butler J, et al. (2010) Prevalence of PALB2 mutations in familial pancreatic cancer. Clin Genet 78(5): 490-494.

16. Roberts NJ, Jiao Y, Yu J, Kopelovich L, Petersen GM, et al. (2012) ATM mutations in patients with hereditary pancreatic cancer. Cancer Discov 2(1): 41- 46.

17. Roberts NJ, Norris AL, Petersen GM, Bondy ML, Brand R, et al. (2016) Whole genome sequencing defines the genetic heterogeneity of familial pancreatic cancer. Cancer Discov 6(2): 166-175.

18. Schneider R, Slater EP, Sina M, Habbe N, Fendrich V, et al. (2011) German national case collection for familial pancreatic cancer $(\mathrm{FaPaCa})-$ ten years experience. Fam Cancer 10(2): 323-330.

19. Bartsch DK, Slater EP, Carrato A, Ibrahim IS, Guillen-Ponce C, et al. (2016) Refinement of screening for familial pancreatic cancer. Gut 65(8): 1314-1321.

20. Langer P, Kann PH, Fendrich V, Habbe N, Schneider M et al. (2009) Five years of prospective screening of high-risk individuals from families with familial pancreatic cancer. Gut 58(10): 1410-1418.
21. Meindl A, Ditsch N, Kast K, Rhiem K, Schmutzler RK, et al. (2011) Hereditary breast and ovarian cancer - new genes, new treatments, new concepts. Dtsch Arztbl Int 108(19): 323-330.

22. Nelson HD, Fu R, Goddard K(2013) Risk assessment, genetic counseling, and genetic testing for BRCA-related cancer: systematic review to update the U.S. Preventive Services Task Force Recommendation [Internet]. Rockville (MD): Agency for Healthcare Research and Quality (US)

23. Goggins M, Overbeek KA., Brand R, Syngal S, Del Chiaro, M, et al. (2020) International Cancer of the Pancreas Screening (CAPS) consortium. Management of patients with increased risk for familial pancreatic cancer: updated recommendations from the International Cancer of the Pancreas Screening (CAPS) Consortium. Gut 69(1): 7-17.

24. Guttmacher AE, Collins FS, Carmona RH (2004) The family history -more important than ever. N Engl J Med 351(22): 2333-2336.

25. Toss A, Venturelli M, Molinaro E, Pipitone S, Barbieri E, et al. (2019) Hereditary pancreatic cancer: a retrospective single-center study of 5143 Italian families with history of BRCA2-related malignancies. Cancers (Basel) 11(2): pp. 193.

26. Igbal J, Ragone A, Lubinski J, Lynch HT, Moller P, et al. (2012) The incidence of pancreatic cancer in BRCA1 and BRCA2 mutation carriers. Br J Cancer 107(12): 2005-2009.

27. Liede A, Karlan BY, Narod SA (2004) Cancer risks for male carriers of germline mutations in BRCA1 or BRCA2. J Clin Oncol 22(4): 735-742.

28. Wang L, Brune KA, Visvanathan K, Laheru D, Herman J, et al. (2009) Elevated cancer mortality in the relatives of patients with pancreatic cancer. Cancer Epidemiol Biomark Prev 18(11): 2829-2834.

29. Hiripi E, Lorenzo-Bermejo J, Li X, Sundquist J, Hemminki K, et al. (2009) Familial association of pancreatic cancer with other malignancies in Swedish families. Br J Cancer 101(10): 1792-1797.

30. Kast K, Rhiem K, Wappemschmidt B, Hahnen E, Hauke J, et al. (2016) Prevalence of BRCA1/2 germline mutations in 21401 families with breast and ovarian cancer. J Med Genet 53(7): 465-471.

31. Greenberg S, Buys SS, Edwards SL, Espinel W, Fraser A, et al. (2019) Population prevalence of individuals meeting criteria for hereditary breast and ovarian cancer testing. Cancer Med 8(15): 6789-6798.

32. Schwartz M, Korenbaum C, Benfoda M, Mary M, Colas C, et al. (2019) Familial pancreatic adenocarcinoma: a retrospective analysis of germline testing in a French multicentre cohort. Clin Genet 96(6): 579584.

33. Abe T, Blackford AL, Tamura K, Ford M, McCormick P, et al. (2019) Deleterious germline mutations are a risk factor for neoplastic progression among high-risk individuals undergoing pancreatic surveillance. J Clin Oncol 37(13): 1070-1080.

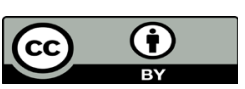

This work is licensed under Creative Commons Attribution 4.0 License

To Submit Your Article Click Here:

Submit Article
DOI: $10.32474 / 0 A J O M .2020 .04 .000178$

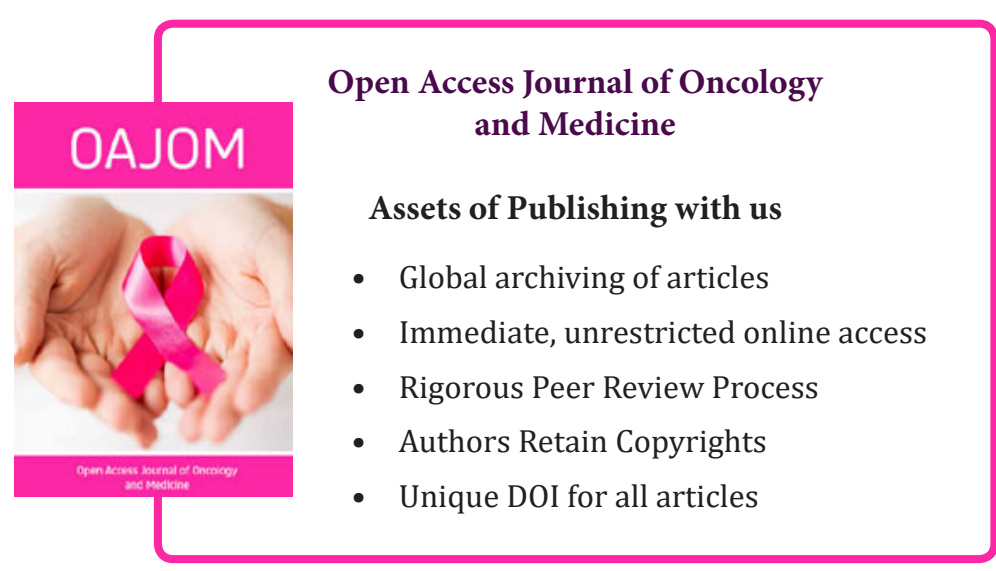

